\title{
Nrf2: new insight in cell apoptosis
}

\author{
M Bonay ${ }^{1,2,3}$ and TB Deramaudt ${ }^{*, 1,3}$ \\ Cell Death and Disease (2015) 6, e1897; doi:10.1038/cddis.2015.256; published online 8 October 2015
}

In a recent issue of Cell Death and Discovery, we described a new mechanism by which sulforaphane decreased mycobacterial burden in an in vitro model of THP-1-derived macrophage infection by Mycobacterium abscessus. ${ }^{1}$ This sulforaphane-mediated diminution was due to the induction of a caspase-independent cell apoptosis and necessitated activation of both nuclear factor E2-related factor 2 (Nrf2) and p38 mitogen-activated protein kinases (MAPK) signaling pathways.

M. abscessus is a nontuberculous mycobacterium that belongs to the group of rapid growing mycobacteria. This emerging pathogen is able to cause skin, bone and soft tissue infections, and more generally exacerbations of lung diseases. ${ }^{2}$ Its high tolerance to antibiotics, which limits patient treatment, has become a public health concern worldwide. M. abscessus has been increasingly involved in patients with cystic fibrosis and in immunosuppressed patients, ${ }^{3}$ enhancing their risk of developing chronic airway infections and leading to a fatal outcome.

Bacterial infection induces an imbalance between oxidants and antioxidants, triggering an oxidative burst that is well known for its bactericidal effect. Yet, some selected mycobacteria are able to thrive in this oxidative environment. It is then unsurprising that the use of oxidant scavengers such as MnTE-2-PyP or N-acetyl-L-cysteine has been shown to decrease $M$. abscessus load by activating bacterial killing in phagolysosomes. ${ }^{4}$ In our study, we decreased the oxidative environment in THP-1-derived macrophages by activating Nrf2, the key transcription factor that controls the cascade of cytoprotective and antioxidant defense mechanisms, and the maintenance of the redox homeostasis. Upon oxidative stress or infection, cytoplasmic Nrf2 is released from its inhibitor Keap-1, translocates into the nucleus, and forms a cofactor complex that binds to specific antioxidant responsive elements (ARE) found in promoters of phase II antioxidant and detoxifying enzymes. Nrf2 signaling pathway has been shown to have an important role whether beneficial or detrimental, in microbial infections. ${ }^{5}$

M. abscessus infection of THP-1-derived macrophages activates Nrf2 signaling pathway, and induces the expression of heme oxygenase-1 and NADPH quinone oxidoreductase-1, two downstream targets of Nrf2. In addition, M. abscessus induced reactive oxygen species (ROS) production in infected macrophages and blocked phagosomal acidification thus allowing its intracellular proliferation.

Sulforaphane, a well-established activator of Nrf2 was used to pretreat macrophages before mycobacterial infection. The results showed an inhibition of mycobacterial growth 7 days postinfection in sulforaphane pretreated macrophages compared with vehicle pretreated ones. This sulforaphaneinduced mycobacterial growth inhibition was neither caused by phagosomal $\mathrm{pH}$ acidification nor increase in cell phagocytosis, but rather induction of cell apoptosis. Contrary to the well-known cyprotective effect of sulforaphane and Nrf2 signaling pathway, ${ }^{6}$ we demonstrated that pre-stimulation of Nrf2 signaling pathway by sulforaphane before $M$. abscessus infection triggered a caspase-independent cell apoptosis. Pretreatment of macrophages with sulforaphane alone had no significant effect on cell death. To our knowledge, this is the first study showing sulforaphane-induced Nrf2 triggers a caspase3/7-independent and p38 MAPKdependent cell apoptosis in mycobacteria infected macrophages (Figure 1).

Cell death in macrophages has a critical role in the host response to mycobacterial infection. Cell apoptosis is an effective mechanism used by the innate defense system to fight bacterial infection. This energy-dependent mechanism generates apoptotic bodies that will facilitate T-dependent antigen response and induce mycobacterial killing by uninfected neighboring macrophages. ${ }^{7}$ As $M$. abscessus has only been recently identified as a separate species, very little is known in the dynamics between $M$. abscessus and macrophage cell survival/cell death. In our in vitro model, $M$. abscessus induced cell necrosis in untreated cells but not cell apoptosis. This phenomenon has been observed in M. tuberculosis that has the ability to inhibit cell apoptosis in favor of cell necrosis. ${ }^{8}$

Recent highlights on caspase-independent forms of apoptosis triggered by infection have been described in the literature. Necroptosis, also known as programmed necrosis, can be initiated by various agents including microbial infection, and is characterized by activation of the receptor-interacting protein (RIP) kinases RIP1 and RIP3-dependent signaling pathway. This programmed cell death is a critical antiviral

\footnotetext{
${ }^{1}$ INSERM U1179, Laboratoire de Physiologie TITAN, UFR des Sciences de la Santé-Simone Veil, Université de Versailles Saint-Quentin-en-Yvelines, Montigny-leBretonneux, France; ${ }^{2}$ Service de Physiologie-Explorations Fonctionnelles, Hôpital Ambroise Paré, Assistance Publique-Hôpitaux de Paris, Boulogne, France and ${ }^{3}$ LIABAHN (Laboratoire International Associé - Biologie Appliquée Handicap Neuromusculaire), CSM (Centre Scientifique de Monaco), Monaco

${ }^{*}$ Corresponding author: TB Deramaudt, INSERM U1179, UFR des Sciences de la Santé-UVSQ, Universite de Versailles, 2, Avenue de la Source de la Bièvre, 78180 Montigny-le-Bretonneux, France. Tel/Fax: +33 170429 415; E-mail: therese.deramaudt@uvsq.fr

Abbreviations: MAPK, mitogen-activated protein kinases; Nrf2, Nuclear factor E2-related factor 2; ROS, reactive oxygen species; TNF, tumor-necrosis factor
} 


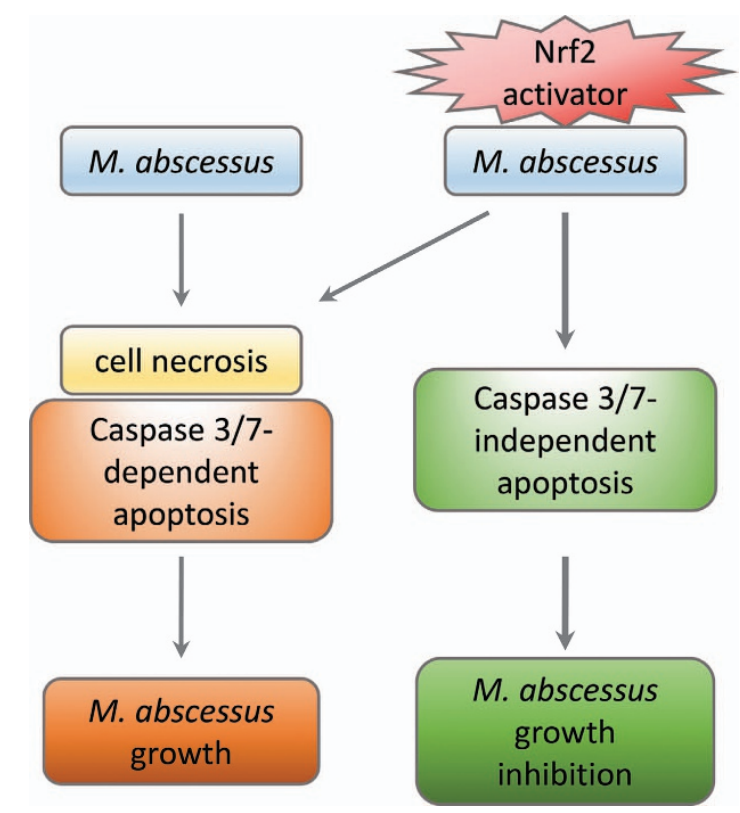

Figure 1 Infection of THP-1 derived macrophages by M. abscessus elicits caspase3/7-dependent cell apoptosis. Pretreatment of macrophages with an Nrf2 activator before mycobacterial challenge initiates a caspase-independent cell apoptosis

process in the host innate immune defense system. Phagocytes infected with viruses that are able to inhibit the classical caspase-dependent apoptosis pathway can undergo necroptosis to facilitate viral clearance by the neighboring immune system. ${ }^{9,10}$ Moreover, Roca and Ramakrishnan have demonstrated a tumor-necrosis factor (TNF)-induced necroptosis in a $M$. tuberculosis infected zebrafish model. In the early stages of mycobacteria infection, injection of an excess of TNF in infected macrophages induced mitochondrial ROS production through a RIP1/ RIP3-dependent kinase pathway, process necessary for the bacterial clearance. However, ROS-mediated necroptosis rapidly led to release and propagation of $M$. tuberculosis in the extracellular microenvironment. ${ }^{11}$

Another caspase $3 / 7$-independent apoptosis is the inflammation-mediated cell death known as pyroptosis. This particular programmed cell death participates in the host cell defense system against pathogens and requires activation of caspase $1, \mathrm{IL}-1 \beta$ and IL-18. ${ }^{12}$ Recently, Bai et al. ${ }^{13}$ have shown that $M$. tuberculosis-mediated production of IL-32 $\gamma$ in THP-1-derived macrophages induced caspase 1-dependent pyroptosis which help reduce intracellular $M$. tuberculosis burden.

In conclusion, additional studies are required to specifically identify the caspase-independent form(s) of apoptosis initiated by the combination of Nrf2 activation and M. abscessus infection. Nevertheless, our findings strongly suggest that Nrf2 activators may be of great interest as future therapeutic treatments in supplement to the actual lengthy multi-drug therapies used in patients diagnosed with mycobacterial infection.

\section{Conflict of Interest}

The authors declare no conflict of interest.

Acknowledgements. This work was supported by funding from the Chancellerie des Universités de Paris (Legs Poix; MB), the Fonds de Dotation Recherche en Santé Respiratoire (MB), the Centre d'Assistance Respiratoire à Domicile d'lle de France (CARDIF; MB), INSERM (TBD, MB), LVL Médical (TBD, MB) and the Bonus qualité recherche-émergence (TBD).

1. Bonay M et al. Cell Death Discov 2015; doi:10.1038/cddiscovery.2015.22.

2. Medjahed H, Gaillard JL, Reyrat JM. Trends Microbiol 2010; 18: 117-123.

3. Orme IM, Ordway DJ. Infect Immun 2014; 82: 3516-3522.

4. Oberley-Deegan RE et al. Free Radic Biol Med 2010; 49: 1666-1673.

5. Deramaudt TB, Dill C, Bonay M. Med Mal Infect 2013; 43: 100-107.

6. Boutten A et al. Trends Mol Med 2011; 17: 363-371.

7. Fratazzi C et al. J Immunol 1997; 158: 4320-4327.

8. Miller JL et al. PLoS Pathog 2010; 6: e1000864.

9. Humphries F et al. Cell Death Differ 2015; 22: 225-236.

10. Sridharan H, Upton JW. Trends Microbiol 2014; 22: 199-207.

11. Roca FJ, Ramakrishnan L. Cell 2013; 153: 521-534.

12. Bergsbaken T, Fink SL, Cookson BT. Nat Rev Microbiol 2009; 7: 99-109.

13. Bai $X$ et al. BMC Microbiol 2015; 15: 39.

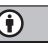

Cell Death and Disease is an open-access journal published by Nature Publishing Group. This work is licensed under a Creative Commons Attribution 4.0 International License. The images or other third party material in this article are included in the article's Creative Commons license, unless indicated otherwise in the credit line; if the material is not included under the Creative Commons license, users will need to obtain permission from the license holder to reproduce the material. To view a copy of this license, visit http://creativecommons.org/licenses/by/4.0/ 